\title{
A Comparative Study of Two English Versions of a Poem in Hong Lou Meng by Yang and Hawkes
}

\author{
Liu Hui ${ }^{1}$ \\ ${ }^{1}$ Ning Xia Normal University, Guyuan, Ningxia,756000
}

Keywords: artistic conception; Three Beauties Principle; comparative analysis

\begin{abstract}
Xu Yuanchong put forward the "Three Beauties Principle-the beauty in sense, sound and form”, which has greatly contributed to translation of Classical Chinese Poetry (CCP). Based on Three Beauties Principle, this paper focuses on the comparison between two versions of the greatest Chinese classic novel Hong Lou Meng, the one translated by Yang Hsien-yi, and the other by David Hawkes. A poem Autumn Window: a Night of Wind and Rain written by Lin Daiyu has been cited to reveal the best faithfulness in poetry translation, and the transference of the artistic conception, the emotion and style of Classical Chinese poetry.
\end{abstract}

\section{Introduction}

Classical Chinese Poetry (CCP) is considered the superior form of Chinese literature. It is characterized by its terseness in language, abstruseness in meaning, sharpness in emotion, and distinctiveness in rhyme and rhythm. It is also known for its artistic conception, which holds a status of significance in Chinese poetry. In some sense, it can be deemed as the essence of Chinese poetry. For this reason, it is a great challenge to translate the classical Chinese poetry into English and to reach artistic conception of original poems. A principle that should be followed in aesthetic requirement is the eternal pursuit in poetic translation. The poem translated must be in possession of three beauties: beauty in sound, beauty in form, and sense. Moreover, aesthetic conception should be made as the starting point of poetic translation. In this article the present writer focuses her attention on a comparative study on two English versions of a poem in the Chinese classical novel Hong Lou Meng (A Dream of Red Mansions), the one by Yang Hsien-yi and Gladys Yang (yang), and the other by David Hawkes and John Minfold (hawkes). Through a comparative study of two versions, the writer reveals the different values and views of the two translators on artistic sound, form, and conception of classical Chinese poetry.

\section{Translation of Classical Chinese Poetry}

\subsection{Characteristics of Classical Chinese Poetry}

Classical Chinese Poetry has long been cherished as the national heritage and the crystallization of Chinese cultures. There are four noticeable characteristics of classical Chinese poetry.

Firstly, classical Chinese poetry is characterized by artistic conception. Artistic conception is the essence and soul of Chinese lyric poetry. As Wang Guowei stated in his book Ren Jian Ci Hua Ci, a genre of Chinese poetry, is deemed the highest in value so long as it has Jingjie. Here, Jingjie refers to the artistic conception. Artistic conception is usually defined as the aesthetic space of imagination generated from a series of images characterized by the fusion of emotion and scenery, including mutual promotion of imagination and reality in the lyrical poems. Accordingly, poetical translation must capture first the artistic mood or the aesthetic conception of the original. In literary translation, artistic conception will appear where emotion is expressed.

Secondly, classical Chinese poetry is marked by its style. According to the Theodore Savory, "Style is the essential characteristic of every piece of writing, the outcome of the writer's personality and his emotions at the moment, and no single paragraph can be put together without revealing in some degree the nature of its author.”(Baldick 2000:137) In the field of poetry, lyrical 
works were composed in the form that derived form the Nine Hymns of the Verse of the Sounth by Qu Yuan, using a six-or seven-character line broken in the middle by the breathing particle "Xi"(兮). Some use lines of irregular length and others are predominantly in five-character lines, which may be said to be forerunners of Tang poetry. As Perine says in his book Sound and Sense, "Poetry is the most condensed and concentrated form of literature, saying most in the fewest number of words." To seek fewest words with the effect of ambiguity and multiplicity of meanings or to seek the most appropriate and graceful-sounding words, poets may make use of the rich connotation of archaic words or allusions to impress the readers indirectly but powerfully. Poem style is much less flexible and less changeable than other literary genres.

Thirdly, classical Chinese poetry is characterized by its figurative language. Figurative language provides a means of achieving color, vividness, subtlety, power and intensity. This particularity of poetry also lies in the poet's arrangement of words, phrases and sentences.

The last but not least important characteristic of poetry is the musicality of poetry. Music, dance, and poetry were originated together from the activities of ancient people. They three were closely knitted together. As time went by, they separated but poetry still cannot go well without music.

\subsection{Translation Principles for CCP}

Before the practical work of translating, translators make some judgment and decisions as to how to translate and on what criteria their translating is to be done. In translating CCP, a well-known translator, $\mathrm{Xu}$ Yuanchong, whose theory is regarded as a perfect goal that all translators who specializing in the CCP translation should follow.

The "Three Beauties" of poetry was first put forward by Xu Yuanchong in the late 1970s. The famous contemporary Chinese translator pioneered a new road both in practice and theory of the translation of CCP. He has rendered large numbers of classical Chinese poems, which called worldwide attention and received highly favorable comments. He summarized "Rendering classical Chinese poems, a translator has to convey the'beauty of sense, beauty of sound and beauty of form'."

The beauty of sense translated poems may touch the hearts of readers just as the original poems did, which is not only the superficial form of the source text, but also the deep meaning. It is of most importance in translating classical poetry.

The beauty of sound means translating rhythm so as to reproduce the beauty in sound of the source text. It is the next importance in translating poetry.

The beauty of form is to maintain original forms as much as possible. It is the least importance.

When possible, all of the three beauties should be well-preserved. As Prof. Xu stated, "In poetry translation, being faithful is the low criterion, whereas, being beautiful is the high one. It is perfect to achieve the unity of faithfulness and beauty. Whereas, when they are contradictory, two choices are available: sacrifice faithfulness for beauty or sacrifice beauty for faithfulness. if the translation is faithful to the original but far less beautiful than the original, it is not worthwhile to sacrifice the beauty; if we could sacrifice faithfulness and obtain beauty, we might say that our gain compensates our loss. Hereby, the translation is better than the original.”

Professor Xu emphasizes that the pursuit of beauty is more important than that of faithfulness and that beauty is the highest aim in poetry translation. To some extent, it is undoubted to say that this theory is a break through to the traditional theories.

Now let's look at a poem translated by two translators, we will use Xu's principles to judge other translator' versions.

\section{A Comparative Study of the Translation Styles of the Two Versions}

It is necessary to introduce the two volume versions of the novel. One of the two English versions of Hong Lou Meng is The Story of the Stone by David Hawkes who translated the former eighty chapters in 1970s. The version is published by Penguin Group. The latter forty chapters were rendered by John Minford. Another version, published by Foreign Languages Press, is entitled $A$ 
Dream of Red Mansions by Yang Hsien-Yi and his wife Dai Naidie in 1970s.

Autume Window: A Night of Wind and Rain in Hong Lou Meng is written by Tsao Hsueh-chin who was a writer in Qing dynasty. The poem is seven-character line in chapter 45 named Two Girls Pledge Friendship After a Heart-to-Heart Talk, A Plaintive Poem Is Written One Windy, Rainy Evening. The poem was written by Lin Daiyu in a windy and rainy night, when she was reading a book named Yuefu Zagao, Inspired by some of the poems, she composed this poem by imitating the pattern of Spring River by the Flower Moon Night, a very famous poem by Zhang Ruoxu in the Tang dynasty.

\section{Yang Hsien-Yi and Dai Naidie}

1 Sad the autumn flowers sear the autumn grass,

2 Autumn lamps flicker through the long autumn night;

3 Unendurable desolate by the autumn window,

4 In the wind and rain autumn seems infinite.

5 The wind and rain speed autumn on its way,

6 By the window shattering her autumn dream;

7 And the girl with autumn in her heart cannot sleep,

8 But trims the candle by her autumn screen.

9 Guttering on its stick, the candle sheds tears of wax,

10 Evoking the grief of separation, its pain,

11 As through each autumn courtyard gusts the wind,

12 And on each autumn window beats the rain.

13 The autumn wind, through silken quilts strikes chill,

14 Her water-clock the autumn rain spurs on

15 All night the pelting rain and soughing wind

16 Accompany her tears for one now gone.

17 Chill mist enwraps the court in loneliness

18 Bamboos drip by the lattice without pause;

19.None can tell when the wind and rain will cease,

20 But already tears have soaked her window's gauze.

\section{David Hawkes}

1 The autumn flowers are dead, the leaves are sere;

2 Lamp-light comes soon, the nights grow long again.

3 Outside my window autumn's sigh appear,

4 More dismal in the wind and rustling rain

5 The rustling rain came in such swift downpour,

6 It startled me from autumn-dream-filled sleep.

7 Now, in a muse, unable to sleep more,

8 I watch the candle at my bedside weep.

9 The candle weeps down to its socket low

10 And my heart weeps and desolation feels.

11 Yet the same wind in other courts must blow;

12 The sound of rain through other windows steals.

13 The wind's chill strikes quilt and counterpane, 14 Thr rain drums like a mad clock in my ears,

15 All night, in whispering, monotone refrain,

16 Companion to my own swift-coursing tears.

17 The courtyard now with mist begins to fill,

18 The bamboo's drip persists without a pause.

19 When will the wind cease and the rain be still,

20 That with its weeping soaks my window's gauze?

\subsection{The Beauty of Sense}

\subsubsection{The Cultural Images of "Autumn" and "Tears"}

The interpretation of poetry starts from the analysis of images. According to the Imagism, images can be divided into "static images" and "dynamic images". Static images refer to nouns, adjectives, and phrases, while dynamic images refer to dynamic description in poems. In original poem, "Autumn" repeats 15 times in the whole poem. Cao Xueqing used this word to describe loneliness, solemn and wretched circumstances. Yang's version keeps the form of the original. It is basically characterized by literal translation, for many of the images are faithfully retained. He renders the static pictures to readers. The typical example is the repetition of the word "autumn" in the translation, but some of them are reasonable and acceptable to Chinese readers, and therefore, achieved the aesthetic effect, such as autumn flowers, autumn grass, autumn night, autumn wind and rain. Some are not natural and less acceptable to the Chinese readers, such as autumn window, autumn screen and "autumn", used in the line "And the girl with autumn in her heart cannot sleep", referring to "qiu qing”. On the contrary, Hawkes' version basically changes the form of the original. It is a dynamic image by using the words of "autumn's signs". In his version, "autumn” is repeated 4 times. Because of this, the original artistic conception is effectively conveyed.

Besides, Hawkes has rendered most of the images. The same image will produce different aesthetic conception if it is translated in different ways. "qiu chuang"is translated literally by Yang into "autumn window", which can give rise to little imagination by readers. On the contrary, Hawkes renders it into "(outside my) window autumn's sigh(appear)," which is not only more acceptable to the readers, but the word "sigh" used here as a homophone to "sign" resulting in an 
effect of pun. Moreover, the fusion of emotion and scenery is achieved.

The language of a nation has a long history, during which it has been branded with national culture. The culture deeply rooted in the language would cause various emotions and associations to the people who use it. For instance, "tear" is a special cultural image in CCP. In the original, the eighth, ninth, sixteenth and twentieth lines: tears, tears of candle, bear very profound and subtle meanings. "tears of candle"is borrowed from Du Mu's poem. Candle is endowed with human feeling and the drips from the burning candle are quite similar to a person's tears. Desolate and gloomy, the poetess loses herself in thought. Watching the burning candle shedding tears of wax, she is suffering from the grief of separation. Hawkes uses different word to express the effect of crying, such as "weep"in line eight, "The candle weeps down"in ninth line and "swift-coursing tears" in the sixteenth line, in the twentieth line, he translates "tears"into weeping soaks. In comparison, in Yang's version, "tears” appear three times: the candle sheds tears of wax, her tears, teas have soaked. In my opinion, yang's translation tries to be absolutely faithful which leads to word-for-word translation that prevents him from expressing the deep sorrow and isolation of poetess.

\subsubsection{Visual Images}

In the original, Cao Xueqing reveals the poetess's inner mind through this poem. The poem is a part of panorama of the novel. Let's compare the two versions; the most obvious distinction between the two versions is the person, which is most likely to be ignored in the translation of classical Chinese poetry. The understanding and disposal of the specific person, however, will lead to different translation effect. The different disposal of this will serve as a good example. Yang's rendering adopts the third person whereas Hawkes' rendering, the first person, like my, me, I, my heart, my ears, my window. It should be noticed that, the first person may well make the reader feel as if he is on the scene on person; moreover, it is more impressive and appealing if we focus our attention on the aesthetic effect. Yang's version may be the result of taking the text as a whole. But it, to some degree, keeps us from entering the artistic conception the poem offers.

\subsubsection{Rhetorical devices}

Rhetorical device is another important stylistic feature of classical Chinese poetry. It is also poses a hard task for all translators. There is the alliteration used in the first line of Yang's translation: "sad" and "sear", which helps to create a picture of the desolate autumn. In the second line, "flicker" is a well-chosen word closely corresponding to the word "耿耿”, which is a pun signifying both the burning of the candle and describes the poetess' gloomy mood. It seems that the second line of Hawkes'version is somewhat not literally in accordance with the original, especially the first half "Lamp-light comes soon" seems to be something added.

The repetition of "autumn" for 15 times in the original creates a sadly moving atmosphere which foreshadows the following delineation. Let's look at the two versions.

\subsection{The Beauty of Sound}

Regarding the beauty of sound in CCP, there are two elements considered: rhyme and repetition of words. The rhyming form of the Yang's version is ABCB, and the last stanza is ABAB. Hawkes' version is rhymed every other line, such as "sere" and "appear" "again" and "rain"; "fill" and "still" "pause" and "gauze". The advantage of it is that the version reads poetic and pleasant both to eye and ear. Yang's version is also rhymed every other line, but some do not rhyme, such as the first stanza. This will inevitably influence the aesthetic effect. Naturalness is needed between lines. Familiarity is necessary in the recreated image. This can make the translated poem natural, fluent, expressive and easy for the readers to accept. So a translator should take into account the impact his translation would have on the readers.

Hawkes'version is delicate in rhyming; especially the rhyme of "sere” and "appear"; "again” and "rain" intensify the poetess's gloom over the autumn. The rhyme in Hawkes' version is in accordance with the original "huang "“chang”"liang” 。 Rhythmically, the four words: “dead”, "sere”, 
"soon" and again", will inevitably be read in falling tone, which is perfectly in agreement with the emotion and spirit of the original. The flaws of this version also lie in one point. For instance, "qiu bu jing"is not conveyed in the third line, yet the clever and creative use of "sign", homophone to sigh, is closely associated with the poetess' innermost feeling. The author's sigh results from the autumn's sign. Hawkes' translation is characterized by its free diction (adding and deleting words), mutation of sentence pattern, hence the strong sense of poetic charm, which also results from the full play of potential of the target language. The most striking example is the rendering of the first two lines in which the punctuation in the middle of each one creates a special effect, reading more like a person's murmuring about something unpleasant. All these help to create the poetic charm. Besides, Hawkes' version employs low-key words, such as weeps, feels, steals, which uses [i:] to rhyme throughout the poem, and down, low, blow - all these help to create a special sound-effect in agreement with the poetess' sad feeling and the artistic mood.

The original poem of over 20 lines with 140 characters is a lyric poem written in a rainy and windy night. It expresses vividly and incisively by means of a series of scenery delineation, the depressed feeling of the poetess separated from her beloved. It mainly consists of seven-character verses. It is original in wording, harmonious in rhythm, concise in style, and pleasant to read.

The repetition of characters, or "die ci" in Chinese, such as "geng geng". Yang adopts word-to-word translation, which is quite similar to the original in the way of expression. What Hawkes pursues is not formal faithfulness. Instead, he pay more attention to the meaning of the poem.

In short, the repetition and the rhyme give the translation a very similar musical way of expression and also the similar effect as the original.

\subsection{The Beauty of Form}

Form is the essential feature of every piece of writing including choice of words, the shape of sentences, the shape of paragraphs, the devices and so on. There are 20 lines in the original text. Both translation versions have 20 lines. It is clear that Hawkes adopts 10-syllabic poem, which is keeping with the original, while Yang avoid a matching of both sound and sense in sentence with the original.

Both versions of the first line change the question into narration while keeping the original meaning. Though the source information is accurately retained, the loss of the original tone can be easily sensed. Yang's version "The wind and rain speed autumn on its way, By the window shattering her autumn dream;" is a faithful transference of the meaning, but the more one reads, the more he feels that it is less poetic, though the lines are rhymed. As for the image "tears of candle", Yang's version "But trims the candle by her autumn screen", though faithfully rendering the activity, fails to transfer the artistic conception. In comparison, Hawkes rendered it into "I watch the candle at my bedside weep" which captured the particular image and conveyed it because the fusion of emotion and scenery can be easily sensed.

\section{Conclusion}

Both versions have their merits and demerits in the transference of the artistic conception of this poem. The important images include: autumn flowers and grass, autumn wind and rain, weeping candle, chill mist, bamboos and window's gauze and so on. All these images are dyed with color of the poetess' depressed feeling and because of this; the poem reads moving and impressive.

Firstly, Yang's version keeps the form of the original. It is basically characterized by literal translation, for many of the images are faithfully retained. The typical example is the repetition of the word "autumn" in the translation, especially the translation of the first two lines of the poem "Sad the autumn flowers, sear the autumn grass, Autumn lamps flicker through the long autumn night" in which not only alliteration is employed, but the words "sad" and "flicker" achieve the fusion of emotion and scenery. But some of them are reasonable and acceptable to Chinese readers, and therefore, achieved the aesthetic effect, such as autumn flowers, autumn grass, autumn night, autumn wind and rain. Some are not natural and less acceptable to the Chinese readers, such as 
autumn window, autumn screen and "autumn", used in the line "And the girl with autumn in her heart cannot sleep", referring to "qiu qing”. On the contrary, Hawkes' version basically changes the form of the original. Because of this, the original artistic conception is effectively conveyed.

Besides, Hawkes has rendered most of the images. The same image will produce different aesthetic effect if it is translated in different ways. "qiu chuang"is translated literally by Yang into "autumn window", which can give rise to little imagination by readers. On the contrary, Hawkes renders it into "(outside my) window autumn's sigh (appear)," which is not only more acceptable to the readers, but the word "sigh" used here as a homophone to "sign" resulting in an effect of pun. Moreover, the fusion of emotion and scenery is achieved.

Secondly, Yang's version is characterized by the translator's tranquil tendency, which familiar with Chinese reader's tastes because of Lin Daiyu's state of melancholy. In comparison, Hawskes' version is characterized by the translator's dynamic role.

Thirdly, the big disadvantage of Yang's version lies in the employment of third person which greatly keeps readers from entering the artistic conception of the poem. Hawkes' version is characterized by the first person, which is closely connected with readers.

Poetry is characterized by its unity of three beauties: beauty in sound, form and artistic conception. As a result, the poem translated must also be in possession of these features. The three beauties of poetry are the common understanding in both Chinese and Western poetics, so it may be adopted as the touchstone to justify the aesthetic effect of poetry translation.

\section{References}

[1] Bassnett, Susan \& Andre Lefevere. Constructiong cultures[M]. Essays on Literary Translation. Shang hai: Shanghai Foreign Language Education Press, 2001

[2] Bonnefoy, Yves. "Translation Poetry”. The Act and the Place of Poetry: Selected Essays[C]. Trans. John Alexander \& Clive Wilmer. The University of Chicago Press, 1989

[3] Catford T.C. A Linguistic Theory of Translation [M]. London: Oxford Univeristity Press, 1965

[4] Cao Xueqin, A Dream in Red Mansions, Beijing: People's Literature Publishing House, 2000.

[5] Feng Qinghua, A Practical Coursebook on Translation, Shanghai: Shanghai Foreign Language Education Press, 2002.

[6] Jin Di, Exploration on Equivalent Transaltion, Beijing: China Translation\& Publishing Corporation, 1998.

[7] Wang Hongyin, Contrastive Study on English Translation of Ancient Poems in A Dream in Red Mansions, Xi'an: Shaanxi Normal University Press, 2001.

[8] Wang Guowei, The Notes and Comments on Ci Poetry, Changchun: Jilin Literature and History Press, 1999.

[9] Xu Yuanchong, The Art of Translation, Beijing: China Translation\& Publishing Corporation, 1984.

[10] Yan Junli, Aesthetic Approach of Poem Translation- Contrastive Study on English Translation of A Poem in A Dream in Red Mansions [D], Shaanxi: Shaanxi Normal University Press, 2003:37-47. 